\title{
An initiative to improve pneumococcal immunization counseling in children with nephrotic syndrome
}

\author{
Ibrahim Sandokji ${ }^{1,2}$ (D) Linda S. Anderson ${ }^{1} \cdot$ Jillian K. Warejko $\cdot$ Beth L. Emerson ${ }^{3} \cdot$ Jason H. Greenberg ${ }^{1}$
}

Received: 6 May 2021 / Revised: 5 September 2021 / Accepted: 7 September 2021 / Published online: 3 November 2021

(c) The Author(s), under exclusive licence to International Pediatric Nephrology Association 2021

\begin{abstract}
Background Immunization is essential in preventing life-threatening pneumococcal infections in children with nephrotic syndrome. An additional 23-valent pneumococcal polysaccharide vaccine (PPSV23) series is required for children with nephrotic syndrome. Despite current practice guidelines, many children with nephrotic syndrome do not receive PPSV23. Methods Our nephrology clinic conducted a quality improvement project to improve the overall rate of PPSV23 counseling to more than $70 \%$ within a 12 -month period by applying several targeted interventions to raise providers' awareness, improve communication with primary care providers, and increase provider adherence. Data was collected from the electronic health record (EHR), and monthly performance was tracked via monthly control charts and overall immunization counseling rate charts.

Results We increased adherence to PPSV23 vaccination counseling from a baseline of 12 to $86 \%$. The first intervention that effectively increased the vaccine counseling rate from 12 to $30 \%$ was improving a provider's awareness of the PPSV 23 literature and vaccine guidelines. Other interventions included regular performance reviews at division meetings, creating an immunization protocol, posting performance charts on the office bulletin board, and unifying vaccine recommendation templates. Lastly, specific and timely EHR reminders improved the total counseling rate from 52 to $86 \%$ and maintained adherence until the completion of the project.

Conclusion Bridging the knowledge gap in provider awareness and using specific EHR reminders can improve adherence to PPSV23 counseling in children with nephrotic syndrome. Such interventions could be applied to similar groups of immunocompromised patients in whom additional vaccines are indicated.
\end{abstract}

Keywords Quality improvement · Immunization · Nephrotic syndrome · Pediatrics · Pneumococcal · Electronic health records

Abbreviations

ACIP Advisory Committee on Immunization Practices

COVID-19 Coronavirus disease

EHR Electronic health record

Ibrahim Sandokji

isandokji@gmail.com

1 Department of Pediatrics, Section of Nephrology, Clinical and Translational Research Accelerator, Yale University School of Medicine, 333 Cedar Street, New Haven, CT 06520-8064, USA

2 Department of Pediatrics, Taibah University College of Medicine, Medina, Saudi Arabia

3 Department of Pediatrics, Section of Pediatric Emergency Medicine, Yale University School of Medicine, New Haven, CT, USA
KDIGO Kidney Disease: Improving Global Outcomes

PCV Pneumococcal conjugate vaccine

PDSA Plan-Do-Study-Act

PPSV23 23-Valent pneumococcal polysaccharide vaccine

SQUIRE Standards for Quality Improvement Reporting Excellence

USRDS United States Renal Data System

\section{Introduction}

Children with nephrotic syndrome are at high risk of lifethreating infections due to hypoalbuminemia, anasarca, urinary losses of immunoglobulins and other proteins, and the use of immunosuppressant medications [1]. Pneumococcal 
peritonitis, bacteremia, and pneumonia are among the leading causes of severe and life-threatening infections in children with nephrotic syndrome [2]. Fortunately, there are several pneumococcal vaccines that help prevent pneumococcal diseases $[1,3]$.

In the USA, most healthy children receive the pneumococcal conjugate vaccine (PCV) series in the first 2 years of life as part of their routine childhood immunization per the Centers for Disease Control and Prevention guidelines [4]. The Advisory Committee on Immunization Practices (ACIP) recommends administering the 23-valent pneumococcal polysaccharide vaccine (PPSV23) to children with nephrotic syndrome older than 2 years of age after completing the routine PCV series [5]. Several studies showed that the pneumococcal vaccine is both safe and effective in children with nephrotic syndrome [3, 6, 7]. Moreover, the Kidney Disease: Improving Global Outcomes (KDIGO) clinical practice guidelines recommend the PPSV23 vaccination of children with nephrotic syndrome, even with concurrent corticosteroid therapy [8]. The PPSV23 immunizes individuals against 23 pneumococcal serotypes and is given in two doses separated by 5 years [5].

Despite the consensus recommendation to administer the PPSV23 in children with nephrotic syndrome, many children do not receive the vaccine [1]. This fact is highlighted in a recent multi-center study demonstrating that rates of PPSV 23 vaccination range between $\sim 6$ and $65 \%$ in children with nephrotic syndrome [9]. Additionally, the 2013 United States Renal Data System (USRDS) annual report showed that only $10 \%$ of children with kidney failure in the USA had received the pneumococcal vaccine despite the recommendation that all children with chronic kidney disease and kidney failure receive the vaccine [10]. The primary reasons for suboptimal PPSV23 vaccination rates are the lack of vaccine counseling by medical providers and lack of knowledge among nephrologists and primary care providers about safety and efficacy of PPSV 23 vaccination in children with nephrotic syndrome [1].

Our overall goal of the present study was to improve rates of PPSV23 vaccination counseling in children with nephrotic syndrome. We hypothesized that by increasing provider knowledge of the PPSV23 research literature and consensus guidelines and improving communication with the primary care provider, as well as improving provider adherence to administer the vaccine, we could increase the number of nephrotic syndrome patients who receive PPSV23 vaccine counseling to more than $70 \%$ within a 12 -month period.

\section{Methods}

To assess our baseline performance, we performed a manual chart review in the electronic health record (EHR) of all children with nephrotic syndrome aged $2-18$ years old in our healthcare practice. During this chart review of the EHR, we searched for a documented PPSV23 administration or counseling done by the treating nephrologist or primary care provider.

\section{Setting}

This project was performed from May 1, 2018, to April 30, 2019, at the pediatric nephrology division at Yale-New Haven Children's Hospital, an academic, tertiary care center in CT, USA. The pediatric nephrology outpatient service consists of six satellite clinics, and each clinic is covered by one of the four board-certified pediatric nephrologists, a registered nurse, and sometimes learners (pediatric nephrology fellows, pediatric residents, and/or medical students). PPSV23 vaccine was not consistently available in all locations.

\section{Measure}

We utilized a process measure of the percentage of eligible patients where PPSV23 vaccine was documented as being recommended. The denominator was defined as the number of nephrotic syndrome patients who did not have a previously documented PPSV23 vaccination or counseling (number of eligible patients). Eligible encounters were clinic visits for eligible patients for that month. PPSV23 vaccination recommendation was sent directly to the primary care physicians (PCPs) through the EHR. For PCPs who do not use the same EHR, the recommendation was faxed to their offices. Once the fax was received, a confirmatory page was printed for documentation. We excluded patients who had a history of severe reaction or documented allergy to pneumococcal vaccine or had moved to another healthcare system. Patients receiving B-cell depleting agents were temporarily removed from the eligible patient list for 6 months after therapy. PPSV23 was recommended after confirmation of adequate pneumococcal 13-valent conjugate vaccination status given the knowledge gaps surrounding recommended pneumococcal vaccination schedule for immunosuppressed children.

\section{Interventions}

This 12-month initiative was planned during multi-disciplinary division meetings. We produced a key driver diagram (Fig. 1) and implemented interventions sequentially [11]. Our interventions were as follows:

1. In month 1, education on the PPSV23 vaccine research literature and the ACIP best practice guidelines was delivered via in-person lectures and follow-up emails 
Fig. 1 Key driver diagram. Multiple interventions were planned and implemented in a sequential manner. Greencolored interventions have been implemented. Grey-colored interventions are ideas to be considered in the future. EHR, electronic health record; PCP, primary care physician
INTERVENTIONS

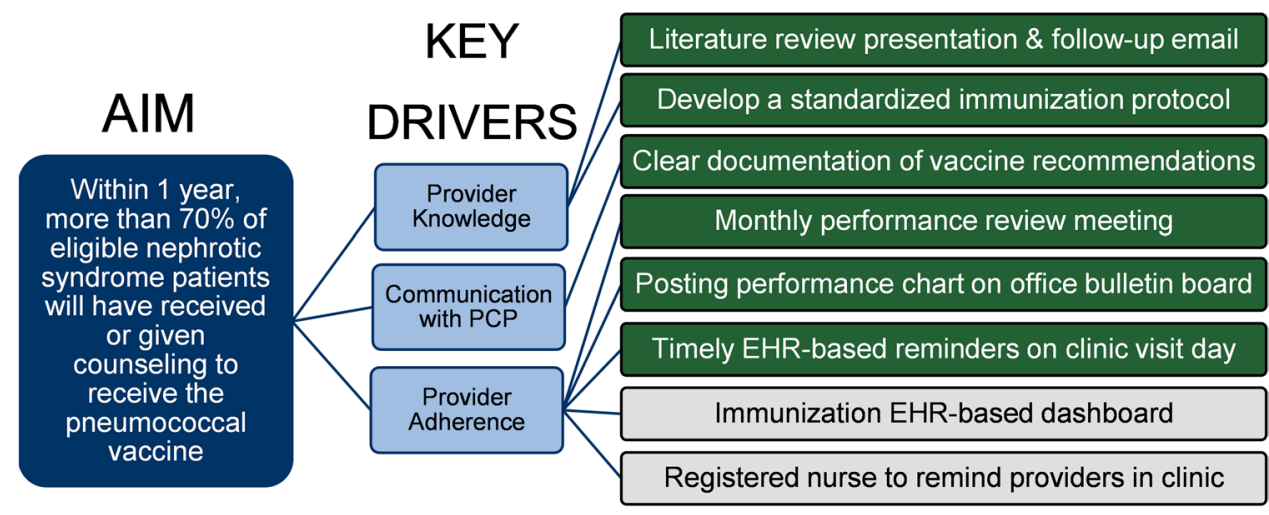

that included key published articles and web links to consensus guidelines. Our web-based presentation can be viewed in Online Resource 1.

2. In month 3 , we added performance reviews to our monthly division meetings.

3. In month 5, we created a divisional immunization protocol using the ACIP recommendations and research literature to standardize the approach to PPSV23 immunization and limit practice variation. The protocol section related to PPSV23 immunization can be viewed in Online Resource 2.

4. In month 6 , we posted performance charts on the division office bulletin board. Those were aggregate data of all clinic providers.

5. In month 7, we utilized a uniform recommendation template in the EHR to communicate with the primary care provider. The template contains the following text: "We recommend administration of the PPSV23 vaccine in the primary care physician's office. This is a two-vaccine series separated by 5 years. Please review immunization records prior to administering the PPSV23. Refer to the $\mathrm{CDC}$ website for pneumococcal vaccine schedule and give as recommended".
6. Lastly, in month 8, we launched EHR-based reminders for eligible patients to arrive in the provider's inbox on the day of an eligible patient's clinic visit. This intervention required a monthly pre-visit chart review, planning for the scheduled visits, and programming the EHR-based reminders in the Epic ${ }^{\circledR}$ EHR system. The EHR-based reminders contain the patient's name and the following message "You are seeing the above patient with nephrotic syndrome today in follow-up. According to our review, the PPSV23 pneumococcal vaccine was not administered in the past. If clinically appropriate, please consider recommending the vaccine and documenting it in your clinic note".

\section{Study of the interventions}

Performance was tracked via control charts to demonstrate the monthly rate of PPSV23 counseling of the eligible encounters with the upper and lower control limits representing 3 SD above and below the mean (Fig. 2). Rules for special cause were followed [12-15]. Additionally, an overall immunization counseling rate chart was created to display the cumulative rate of eligible patients who received
Fig. 2 Control chart of monthly vaccination counseling rate. Different interventions led to variable degrees of improvement in PPSV23 monthly counseling rates. The center line was shifted at month 8 , when timely EHR-based reminders were initiated. The numerator is the number of eligible patients who received counseling (or vaccination) in each month. The denominator is the total number of eligible patients seen in clinic in that month. CL, center line; LCL, lower control limit; UCL, upper control limit

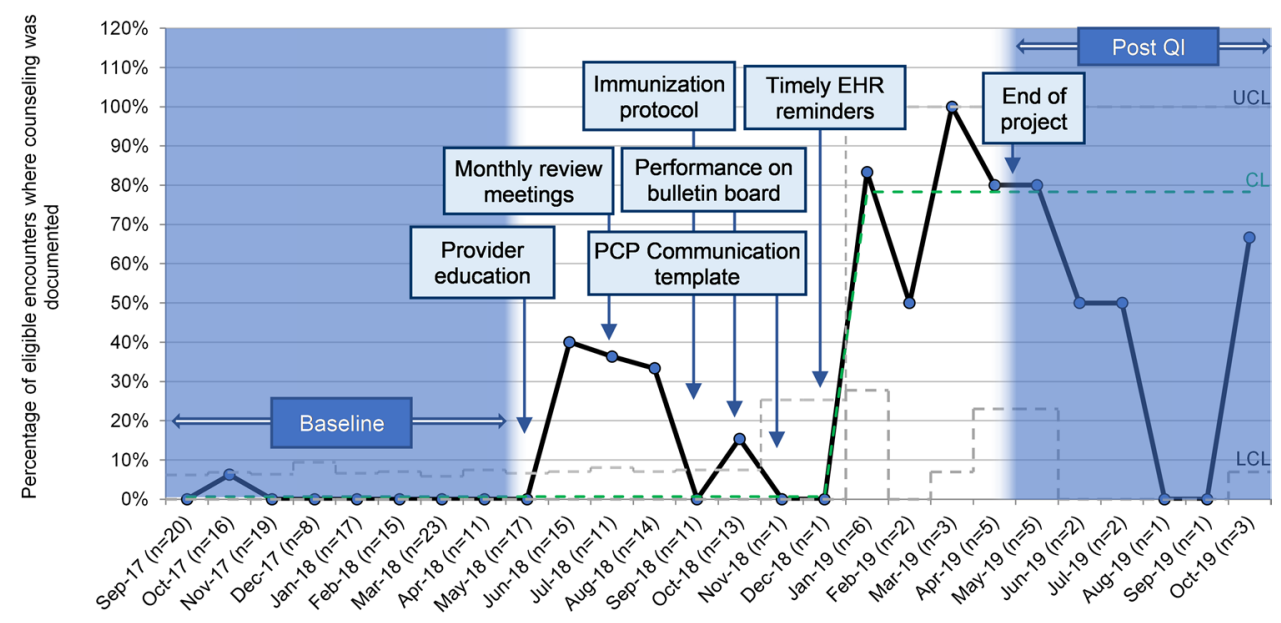

Eligible encounters in each month 
PPSV23 counseling out of the total current number of children with nephrotic syndrome in our practice (Fig. 3). Control charts were created using QI Macros for Excel (KnowWare International, Denver, CO). Data was collected from the EHR.

\section{Ethical considerations}

This quality improvement project was exempted from review per institutional review board regulations. We report our project in compliance with the Standards for Quality Improvement Reporting Excellence (SQUIRE 2.0) guidelines (Online Resource 3) [16].

\section{Results}

At baseline, our center cared for a total of 68 children with nephrotic syndrome. The median age of these 68 patients at the start of this quality improvement project was 10 years (IQR, 6-14) and 36 (48\%) were male. Eight out of the 68 children $(12 \%)$ had either received the recommended PPSV23 vaccine or were advised by the treating nephrologist to receive it at the primary care physician's office.

To reach our goal, three key drivers were defined: provider knowledge, communication with the primary care physician, and provider adherence (Fig. 1). Figure 2 is a control chart that illustrates the monthly vaccination counseling rate. The first intervention was focused on educating medical providers on the PPSV23 research literature and consensus guidelines $[1,3,5-7]$. This intervention increased the overall counseling rate from the baseline of 12 to $30 \%$ (Fig. 3). Next, monthly review meetings of the PPSV 23 counseling rates were initiated from month 3 and increased the counseling rate to $44 \%$. Then, in month 5 , the creation of a division immunization protocol improved the counseling rate to $48 \%$. In month 6 , we posted PPSV23 counseling performance charts on the office bulletin board, and the vaccination counseling rate continued to rise to $50 \%$. The use of a uniform EHR template for PPSV23 communication with the primary care provider helped to standardize the communication process and increased the rate of counseling to $52 \%$.

We observed a decrease in counseling during the months of November and December 2018. However, during that period, there were only two patients with nephrotic syndrome seen in clinic due to inclement weather and holidayrelated clinic closures.

Lastly, the EHR-based provider reminders were implemented in month 8 and led to a sustained improvement in performance for the remaining months of the project during which the quality improvement project goal was achieved. The center line of the control chart was shifted due to the presence of six consecutive points above the preceding center line (Fig. 2). By the end of the project, we observed that $86 \%$ of children had received PPSV23 counseling (Fig. 3). Later, after completion of the 12-month project and cessation of the monthly review meetings and EHR-based alerts, we observed a decrease in the monthly counseling rate to approximately $50 \%$.

\section{Discussion}

We successfully implemented a multi-faceted quality improvement initiative to increase the rate of PPSV23 counseling in children with nephrotic syndrome. This project led to an increase in the percent of children who received vaccination counseling from 12 to over $80 \%$ by the end of the project.

To our knowledge, this is the first reported quality improvement project focused on improving PPSV23 immunization in children with nephrotic syndrome. Several quality improvement initiatives have been previously reported to improve immunization in immunocompromised populations.
Fig. 3 Overall cumulative vaccination counseling rate. Several interventions were implemented. The quality improvement project's specific aim was to reach more than a $70 \%$ counseling rate of eligible nephrotic syndrome patients within a 12-month period and this aim was achieved in month 11 . The numerator is the cumulative total number of eligible patients who received counseling (or vaccination). The denominator is the cumulative total number of eligible patients actively followed in our clinics

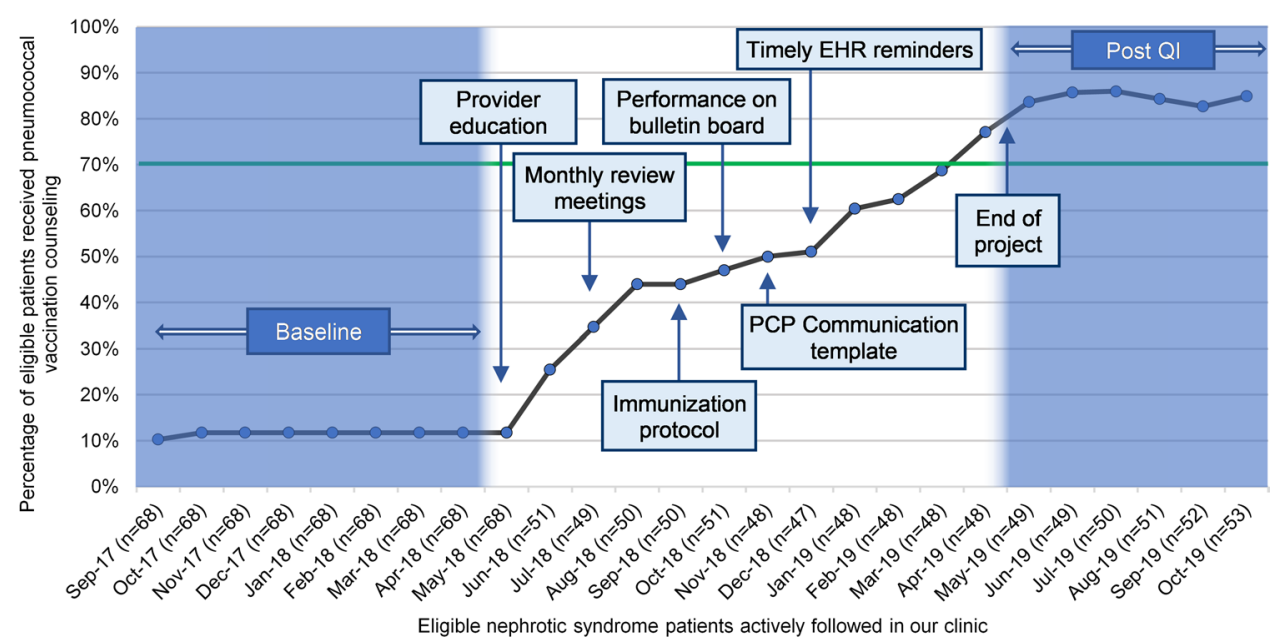

Eligible nephrotic syndrome patients actively followed in our clinic 
Malone et al. reported a multi-faceted quality improvement project that successfully improved pneumococcal immunization rates in children after kidney transplantation from 6 to $52 \%$ by developing an age-specific vaccine algorithm, obtaining vaccine records from primary care providers, and using EHR-based best practice advisory reminders [17]. Another quality improvement initiative to increase pneumococcal vaccination in children with systemic lupus erythematosus was performed over a 12-month period with several interventions that included increasing provider knowledge, creating an immunization algorithm, pre-visit planning, and EHR-based reminders [18]. Furthermore, a recent 12-month quality improvement project improved the percentage of children with systemic lupus erythematosus who received at least one pneumococcal vaccine from $\sim 25$ to more than 90\% using interventions that increased provider awareness of the PPSV23 vaccine, routine review of patient vaccine records, weekly pre-visit planning, and creating a vaccine algorithm [19].

In the present project, we noted an improvement of the PPSV23 counseling rate beginning when educational programs for providers were initiated. Education helps to improve medical provider understanding of the correct timing, dosing, efficacy, and possible side effects of PPSV23 vaccination in children with nephrotic syndrome. In a recent multi-center study of 50 pediatric nephrologists and 153 parents of children with nephrotic syndrome, only $44 \%$ of providers reported adherence to the inactive vaccine guideline recommendations, and $32 \%$ of the parents reported awareness of these recommendations [9].

We focused on provider counseling as a process measure due to the significant impact of counseling on the parental decision for immunization. Tran et al. examined the predictors of PPSV23 adherence in children with nephrotic syndrome and found that a recommendation for immunization by the treating nephrologist was significantly associated with vaccine adherence [9]. This association was maintained in a logistic regression model adjusted for several factors including parent income, parent education, and race. Furthermore, Scheuerman et al. reported that the most important reason for parents of children with chronic kidney disease who did not accept influenza vaccination was not receiving appropriate counseling and $38 \%$ of the parents stated they would have agreed if they received sufficient information [20].

With the initiation of EHR-based reminders, we observed a sustained improvement in adherence to PPSV23 counseling. Providers receiving patient-specific alerts on the morning of a clinic visit were more likely to counsel their patients and parents during busy clinic days. This is highlighted by the shift in the center line of the control chart starting from month 8 . Several quality improvement initiatives leveraged timely reminders to improve immunization compliance $[17,18]$. The use of EHR-based tools to modify provider behaviors can be a double-edged sword. In a quality improvement project, Malone et al. reported no improvement in vaccination compliance with using non-specific EHRbased alerts that were delivered to all providers seeing kidney transplant patients [17]. Alternatively, we used specific reminders to individual patients that arrived at the assigned provider's inbox on the morning of clinic visits to avoid "alarm fatigue". These alerts were generally well-tolerated and encouraged by providers, as evidenced by feedback during division meetings and the sustained improvement in counseling rates. Of note, the weekly chart review and EHR alerts pre-programming took between 30 and $45 \mathrm{~min}$ initially, but decreased with time as fewer patients were eligible. This is consistent with similar projects using preprogrammed EHR alerts [19].

Our project has several limitations. This is a single center effort which may limit generalizability. Also, we relied on the PCPs to administer the vaccination due to the unavailability of the PPSV23 vaccine in many of our satellite clinics and to enhance the role of the PCPs in centrally managing and tracking vaccinations. Our focus on PPSV 23 counseling, as opposed to delivery of the vaccine, was due to the lack of PPSV23 vaccines at many pediatric nephrology satellite clinics and the PCP's access to detailed historical vaccination records. Also, in this project, we focused on the process measure of counseling parents and patients, but we did not assess if the actual vaccination was administered by the PCPs. This can be included in future projects to ensure the receipt of the recommended vaccination. We also recognize the overlap between consecutive intervention cycles, which should be considered in evaluating the impact of each intervention cycle. Additionally, after our EHR reminders and division meetings stopped, we witnessed a decline in counseling rates as PPSV23 vaccination was not discussed among providers as frequently. Future directions should include sustainable improvement plans such as pre-programmed EHRbased alerts and routine discussion at division meetings. We included the performance of the post-QI period for complete transparency and to encourage others to study strategies to maximize adherence in the post-QI period.

Several of our findings may be generalizable to other subspecialists taking care of immunocompromised children. In our center, bridging knowledge gaps with educational programs and open discussions about consensus guidelines was an important first step to improving vaccination counseling rates. An EHR-based reminder can lead to an improvement in provider compliance. These alerts must be specific and timely to achieve the desired quality improvement and avoid "alarm fatigue".

In conclusion, we were able to increase the total immunization counseling rate from $12 \%$ at baseline to more than $80 \%$ by the end of a 12 -month period, using sequential interventions. Such interventions can be applied to other 
populations, such as other immunosuppressed children and those with chronic kidney disease, and may further prevent severe illness due to preventable infections.

Supplementary Information The online version contains supplementary material available at https://doi.org/10.1007/s00467-021-05305-3.

Acknowledgements The authors would like to thank all of the pediatric nephrology clinic providers and nurses at Yale-New Haven Children's Hospital, in particular Julie Goodwin, MD; Amirtha V. Chinnadurai, MD; Marie Campbell; Juliann Reardon, MD; and Olivera M. Couloures, MD.

Author contribution Ibrahim Sandokji conceptualized and designed the study, collected data, carried out the initial analyses, drafted the initial manuscript, made the figures, and reviewed and revised the manuscript. Linda S. Anderson collected data and reviewed and revised the manuscript. Beth L. Emerson supervised data collection and critically reviewed the manuscript and figures for important intellectual content. Jason H. Greenberg and Jillian K. Warejko conceptualized and designed the study, supervised data collection, and critically reviewed the manuscript for important intellectual content. All authors approved the final manuscript as submitted and agree to be accountable for all aspects of the work.

\section{Declarations}

Conflict of interest The authors declare no competing interests.

\section{References}

1. Goonewardene ST, Tang C, Tan LT, Chan KG, Lingham P, Lee LH, Goh BH, Pusparajah P (2019) Safety and efficacy of pneumococcal vaccination in pediatric nephrotic syndrome. Front Pediatr 7:339

2. Uncu N, Bulbul M, Yildiz N, Noyan A, Kosan C, Kavukcu S, Caliskan S, Gunduz Z, Besbas N, Gur Guven A (2010) Primary peritonitis in children with nephrotic syndrome: results of a 5-year multicenter study. Eur J Pediatr 169:73-76

3. Vandecasteele SJ, Ombelet S, Blumental S, Peetermans WE (2015) The ABC of pneumococcal infections and vaccination in patients with chronic kidney disease. Clin Kidney J 8:318-324

4. Centers for Disease Control and Prevention (2019) Vaccination coverage for selected diseases by age 24 months, by race and Hispanic origin, poverty level, and location of residence: United States, birth years 2010-2015. https://www.cdc.gov/nchs/data/ hus/2019/031-508.pdf. Accessed 27 Sept 2021

5. Nuorti JP, Whitney CG (2010) Prevention of pneumococcal disease among infants and children - use of 13-valent pneumococcal conjugate vaccine and 23 -valent pneumococcal polysaccharide vaccine - recommendations of the Advisory Committee on Immunization Practices (ACIP). MMWR Recomm Rep 59:1-18

6. Liakou CD, Askiti V, Mitsioni A, Stefanidis CJ, Theodoridou MC, Spoulou VI (2014) Safety and immunogenicity of booster immunization with 7-valent pneumococcal conjugate vaccine in children with idiopathic nephrotic syndrome. Vaccine 32:1394-1397
7. Pittet LF, Posfay-Barbe KM, Chehade H, Rudin C, WilhelmBals A, Rodriguez M, Siegrist CA, Parvex P (2016) Optimizing seroprotection against pneumococcus in children with nephrotic syndrome using the 13-valent pneumococcal conjugate vaccine. Vaccine 34:4948-4954

8. Cattran DC, Feehally J, Cook HT, Liu ZH, Fervenza F, Mezzano S, Floege J, Nachman PH, Gipson DS, Praga M,Glassock R, Radhakrishnan J, Hodson E, Rovin B, Jha V, Troyanov S, Li PKT, Wetzels J (2012) Kidney disease:Improving global outcomes (KDIGO) glomerulonephritis work group. KDIGO clinical practice guideline forglomerulonephritis. Kidney International Supplements 2:139-274

9. Tran CL, Selewski DT, Oh GJ, Troost JP, Massengill SF, AlAkash SI, Mahesh S, Amin R, Ashoor IF, Chanchlani R, Kallash M, Woroniecki RP, Gipson DS (2021) Pediatric immunization practices in nephrotic syndrome: an assessment of provider and parental knowledge. Front Pediatr 8:619548

10. National Institutes of Health (2013) 2013 USRDS annual data report: atlas of chronic kidney disease and end-stage renal disease in the United States. https://www.usrds.org/media/1485/v2_00_ intro_13.pdf. Accessed 27 Sept 2021

11. Gaudreault-Tremblay MM, McQuillan RF, Parekh RS, Noone D (2020) Quality improvement in pediatric nephrology-a practical guide. Pediatr Nephrol 35:199-211

12. (2017) Quality, service improvement and redesign tools: statistical process control (SPC). https://www.england.nhs.uk/sustainabl eimprovement/qsir-programme/qsir-tools/. Accessed 27 Sept 2021

13. Oakland JS (2007) Statistical process control. Routledge, Abingdon

14. Kenett RS, Baker E (1999) Software process quality: management and control. CRC Press, Boca Raton

15. Perla RJ, Provost LP, Murray SK (2011) The run chart: a simple analytical tool for learning from variation in healthcare processes. BMJ Qual Saf 20:46-51

16. Ogrinc G, Davies L, Goodman D, Batalden P, Davidoff F, Stevens D (2016) SQUIRE 2.0 (standards for quality improvement reporting excellence): revised publication guidelines from a detailed consensus process. BMJ Qual Saf 25:986-992

17. Malone K, Clark S, Palmer JA, Lopez S, Pradhan M, Furth S, Kim J, Fisher B, Laskin B (2016) A quality improvement initiative to increase pneumococcal vaccination coverage among children after kidney transplant. Pediatr Transplant 20:783-789

18. Harris JG, Maletta KI, Ren B, Olson JC (2015) Improving pneumococcal vaccination in pediatric rheumatology patients. Pediatrics 136:e681-686

19. Sivaraman V, Wise KA, Cotton W, Barbar-Smiley F, Al Ahmed O, MacDonald D, Lemle S, Yildirim-Toruner C, Ardoin SP, Ardura MI (2020) Previsit planning improves pneumococcal vaccination rates in childhood-onset SLE. Pediatrics 145:e20183141

20. Scheuerman O, Zilber E, Davidovits M, Chodick G, Levy I (2017) Nephrologists need to play a key role in improving annual influenza vaccination rates in children with kidney disease. Acta Paediatr 106:812-818

Publisher's note Springer Nature remains neutral with regard to jurisdictional claims in published maps and institutional affiliations. 